\title{
SELEÇÃO DAS ESTRUTURAS CALCIFICADAS PARA A DETERMINAÇÃO DA IDADE DA PIRACATINGA Calophysus macropterus Lichtenstein (Siluriformes: Pimelodidae) NA AMAZÔNIA CENTRAL, BRASIL.
}

\author{
Alfredo PÉREZ1 \& Nidia Noemi FABRÉ ${ }^{2}$
}

\begin{abstract}
RESUMO - O objetivo deste trabalho foi identificar a estrutura calcificada mais adequada para a determinação da idade da piracatinga (Calophysus macropterus). Foram analisados 440 indivíduos procedentes da pesca comercial e experimental do Baixo Solimões. Deste total, foram analisados opérculos, otólitos (asteriscus) e vértebras de uma sub-amostra de 130 indivíduos. Para a observação dos anéis de crescimento nos otólitos, foram testadas duas técnicas de clarificação e quatro técnicas de coloração nas vértebras. A análise das estruturas calcificada demonstrou que os otólitos são estruturas pouco translúcidas e apresentam dificuldades para a observação das marcas de crescimento. Por outro lado os opérculos não apresentaram marcas visíveis de crescimento, e nas vértebras foi observado um padrão nítido e estável de marcação de anéis de crescimento. Portanto, as vértebras foram as estruturas mais adequadas para determinar a idade da piracatinga, além de apresentar uma relação linear significativa entre o raio total das vértebras e o comprimento do peixe $(r=0,91 ; \mathrm{P}<0,05)$. Entre os métodos testados para a visualização dos anéis nas vértebras de peixes teleosteos, a técnica nova a de Hiper-oxidação e Descalcificação Química (HDQ), mostrou ser a melhor. As marcas de crescimentos em vértebras foram validadas utilizando o método de incremento marginal relativo. A piracatinga forma duas marcas de crescimento por ano nas vértebras; o primeiro durante a vazante (agosto) e o segundo durante a enchente (janeiro).
\end{abstract}

Palavras-chave: Otólitos, opérculos, vértebras, Calophysus macropterus, rio Solimões,

\section{Selection of Calcificated Structure for Aging of the Piracatinga, Calophysus macropterus Lichtenstein (Siluriformes: Pimelodidae) in the Central Amazon, Brazil.}

\begin{abstract}
The goal of this study was to identify the best calcified structure for determination of the age of the piracatinga (Calophysus macropterus). A total of 440 specimens from commercial samples and experimental fishing in the Solimões River. Were used for analysis: Opercula, otoliths, and vertebrae were analyzed from a sub sample $(\mathrm{n}=130)$. Two clarification techniques were used for visualization of growth rings in the otoliths and four coloration techniques for vertebrae. The otoliths were not translucent and it was very difficult to see the growth rings. The vertebrae were the best calcified structure because the radius of the vertebrae and the length of fish had clear significant linear relationship $(\mathrm{r}=0.91 ; \mathrm{P}<0.05)$ and a clear visualization of the growth rings. Among the techniques used for examining vertebrae of the teleostean fishes, the chemical decalcification, hyper-oxidation technique, was the best to observe the growth rings in the vertebrae. The seasonal rings were validated using relative marginal incremental analysis. Two annual rings are formed in the vertebrae of Calophysus macropterus, being the first during low water (August) and the second during high water season (January). On the vertebrae, a maximum of seven rings were observed.
\end{abstract}

Key-words: Otoliths, opercula, vertebrae, Calophysus macropterus, Solimões river

1Vice-rectorado de Planificación y Desarrollo Regional, Apartado Postal \# 04, San Fernando, 7001. Estado Apure, Venezuela. e-mail: aperez@reacciun.ve

${ }^{2}$ Laboratório de Ecologia, Instituto de Ciências Biológicas, Universidade do

Amazonas, Mini-campus. Manaus-AM, Brasil. e-mail: tchoni1@uol.com.br 


\section{INTRODUÇÃO}

A determinação da idade por meio de estruturas calcificadas vem sendo amplamente utilizada nas ciências pesqueiras desde o início do século XX. Sendo as escamas e otólitos as estruturas calcificadas mais utilizadas para a determinação da idade em peixes teleósteos.

Em qualquer estudo para a determinação da idade usando estruturas calcificadas, devemos discriminar as marcas de crescimento sazonal verdadeiras das falsas (Nikolsky, 1969), testando técnicas para sua melhor visualização. Metodologicamente é recomendável analisar diferentes estruturas calcificadas utilizando mais de um procedimento de tratamentos independentes e diferentes (Brothers, 1983).

Segundo Brothers (op. Cit.), a verificação constitui na observação das marcas de crescimento em diferentes estruturas calcificadas de peixes, aplicando simultaneamente diferentes métodos de visualização de marcas.

Porém, o principal problema com os métodos de verificação é que dependem das técnicas utilizadas na interpretação da idade. Portanto, os critérios específicos para o reconhecimento das marcas de crescimento deveriam ser feitos para que os erros associados com a interpretação pudessem ser avaliados (Casselman,1983).

Vários trabalhos foram realizados na Amazônia para a determinação da idade em peixes comerciais (Werder \& Soares, 1985; Villacorta-Corrêa, 1987, 1997; Panfili, 1993; Oliveira,1997; Fabre \& Saint-Paul, 1998; Vieira, 1999). Porém, no caso dos peixes Siluriformes são poucos os estudos sobre a idade utilizando estruturas calcificadas, sendo os otólitos, vértebras e espinhos peitorais as estruturas mais utilizadas (Reid, 1983; Barthem,1990; Panfili, 1993; Reina et al. 1995; Muñoz-Sosa, 1996; Pérez, 1999).

Fabré et al.(1995), testaram em um estudo com Calophysus macropterus na Amazônia Central, a eficiência da técnica de La Marca (1966) em vértebras desta espécie e observaram anéis fortemente marcados, geralmente duplos, alternando-se com marcas intermediárias nem sempre constantes, o que dificulta a interpretação das marcas anuais, porém, devido ao baixo número de observações o trabalho não foi conclusivo.

Apesar da grande importância econômica de algumas espécies de bagres na região Amazônica, ainda não existe uma metodologia padronizada para a determinação da idade utilizando estruturas calcificadas. Por outro lado, a maioria das espécies comerciais de bagres são migratórias, o que dificulta a obtenção das amostras durante um ciclo completo. Portanto, devido à facilidade de realizar uma amostragem continua durante um ciclo hidrológico, a piracatinga foi selecionada como espécie padrão para este estudo.

Assim, os objetivos deste trabalho foram: 1)Testar diferentes técnicas de visualização de anéis em estruturas calcificadas para selecionar a mais adequada, visando estudos de determinação da idade na piracatinga, 2) Descrever e avaliar a nitidez e a estabilidade do padrão de marcação dos anéis na estrutura calcificada selecionada e 3) Determinar a periodicidade de formação das marcas de crescimento com a estrutura calcificada selecionada.

\section{MATERIAIS E MÉTODOS}

\section{Coleta e preparação do material}

Os peixes foram coletados mensalmente durante um ano totalizando 440 exemplares provenientes da pesca experimental no lago Catalão ( $3^{\circ} 10^{\prime} \mathrm{S} ; 59^{\circ} 55^{\prime} \mathrm{W}$ ) no período de junho a agosto de 1997 e da pesca comercial praticada no município de Iranduba, rio Solimões ( $3^{\circ} 17^{\prime}$ $\mathrm{S} ; 60^{\circ} 20^{\prime} \mathrm{W}$ ) de setembro de 1997 a maio de1998 (Figura 1).

Para a escolha da melhor estrutura na determinação da idade, utilizou-se uma subamostra de 130 indivíduos de diferentes classes de comprimentos padrão, entre $(10$ e $40 \mathrm{~cm})$. De cada exemplar foram registrados dados referentes ao sexo, comprimentos furcal e padrão $(\mathrm{cm})$. 


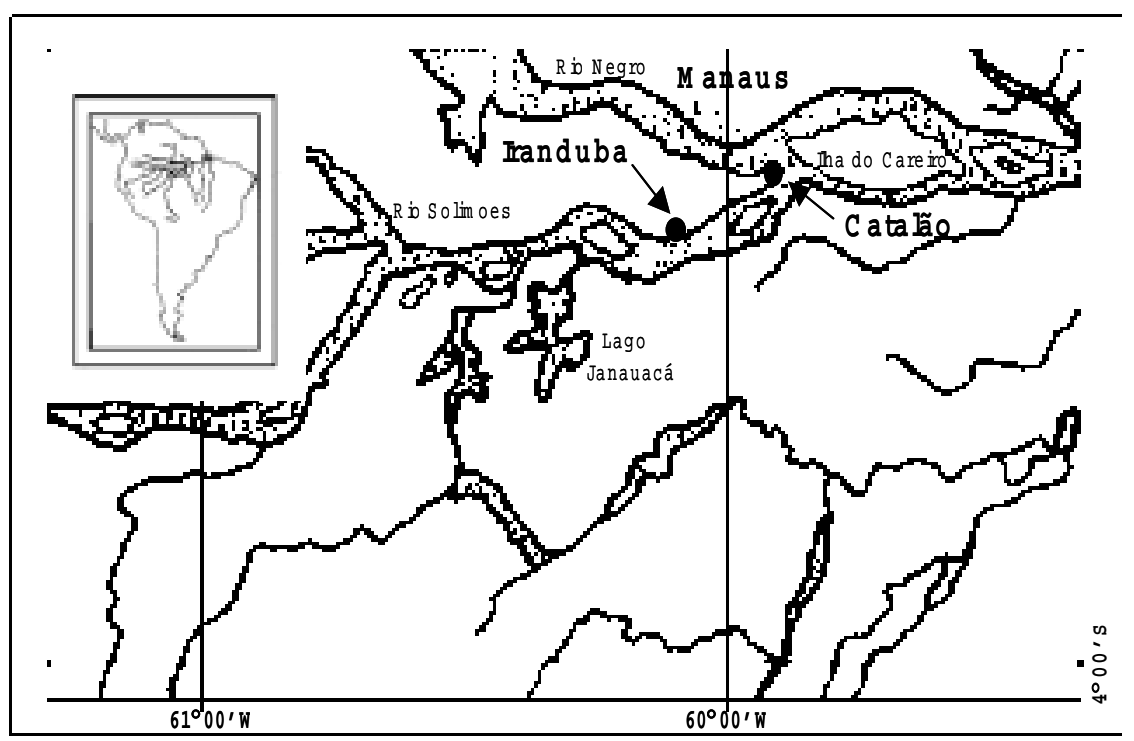

Figura 1. Áreas de coleta de Calophysus macropterus, Iranduba e Catalão (Amazônia Central) representadas por círculos negros.

\section{Opéculos}

Foram analisados os opérculos de 15 exemplares de peixes com comprimento padrão variando entre 10 e $35 \mathrm{~cm}$. Os opérculos foram retirados do osso hiomandibular e limpos utilizando água quente por 5 minutos para eliminar a maior quantidade possível de tecido muscular e adiposo. No tratamento para a observação e contagem das marcas de crescimento foram utilizadas as técnicas de La Marca (1966) e Daiber (1960).

As leituras das estruturas calcificadas tratadas com as diferentes técnicas, foram realizadas com o auxílio de um Microscópio estereoscópio Zeiss modelo STEMI SV 6, com micrômetro ocular, acoplado a uma câmara de vídeo JVC KT1180U, ligada a um monitor JVC de discussão. Foi utilizada uma fonte de luz fria refletida Zeiss KL 1500 electronic. Todas as estruturas calcificadas foram observadas submersas em uma solução de álcool etílico P.A. a 70\%, em um recipiente de bakelita preto com uma concavidade de fundo.

\section{Otólitos}

Cento e onze pares dos otólitos (lapillus e asteriscus) foram coletados com o auxilio de um bisturi, através de um corte frontal na metade do osso paraesfenoide, até a sínfisis com o osso basioccipital. $\mathrm{O}$ otólito sagitta não foi coletada devido a sua fragilidade e pela dificuldade de observação dos anéis de crescimento

Para estabelecer qual substância facilita a melhor visualização das marcas de crescimento, os otólitos foram clarificados com xilol e benzoato de metil. O otólito lapillus não foi utilizado neste estudo, por tratar-se de uma estrutura opaca e por não apresentar marcas de crescimento visíveis. Como critérios de avaliação para a seleção dos otólitos, foram utilizados: nitidez das marcas de crescimento, tipos de marcas e padrão de espaçamento entre elas.

Para a análise morfométrica, foram registradas as seguintes medidas no otólito asteriscus em unidades micrométricas $(\mu \mathrm{m})$ : largura máxima (LM), raio dorsal (RD), comprimento máximo $(\mathrm{CM})$ e raio total $(\mathrm{Rt})$ (Figura 2). 


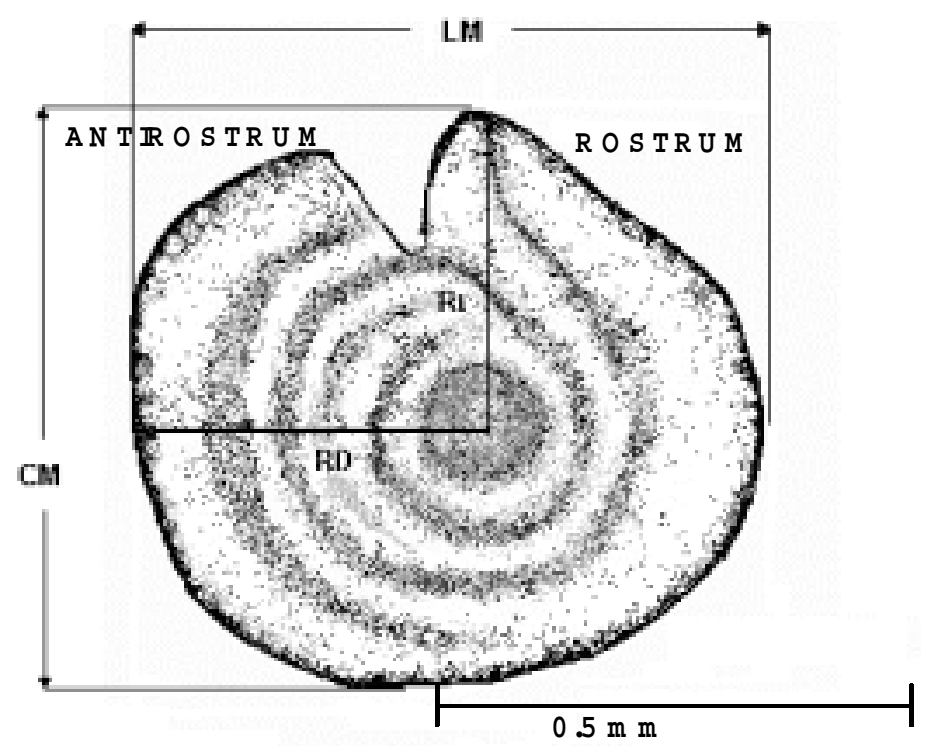

Figura 2. Esquema da face externa do otólito asteriscus, indicando as medidas obtidas nesta estrutura, (LM: largura máxima; CM: comprimento máximo; RD: raio dorsal; Rt: raio total).

\section{Vértebras}

Da porção mediana da coluna vertebral posicionada entre o final da nadadeira dorsal e no primeiro terço anterior da nadadeira adiposa (vértebras N. $\left.{ }^{\circ} 10-19\right)$, foram retiradas de 4 a 5 vértebras e em seguida, colocadas em água quente por 5 minutos para eliminar a maior quantidade possível de tecido muscular e adiposo.

Para observar as marcas de crescimento nas vértebras, foram testadas as técnicas de Daiber (1960), La Marca (1966), Officer et al., (1995) e uma nova técnica denominada Hiperoxidação e Descalcificação Química (HDQ) (Figura 3).

A técnica de Hiper-oxidação e Descalcificação Química, caracteriza-se pela aplicação de um processo duplo de extração das gorduras (hiper-oxidação) e de hidratação da estrutura antes da coloração, utilizando o azul de toluidina como corante devido a alta afinidade com as proteínas estruturais o que facilita a visualização das marcas de crescimento.
Para determinar qual é a melhor técnica para visualizar as marcas de crescimento, foram selecionadas 24 vértebras, sendo utilizadas quatro para cada técnica, além do controle. No caso da técnica de Hiper-oxidação e Descalcificação Química foram utilizadas oito vértebras, quatro para cada corante.

Para selecionar na seleção da melhor técnica de coloração ou clarificação das vértebras, foram considerados os seguintes critérios: nitidez das marcas, tipo de marcas, presença de marcas intermediárias e o padrão de espaçamento entre elas. Para a análise morfométrica, foi registrado o raio total (Rt) a $45^{\circ}$ expressos em unidades micrométricas $(\mu \mathrm{m})$.

Uma vez selecionada a melhor estrutura calcificada, a normalidade das distancias de cada marca de crescimento foi verificada utilizando o teste Kolmogorov-Smirnov para normalidade entre grupos (Sokal \& Rolf, 1981).

Para avaliar a relação entre o comprimento padrão e o raio total da estrutura calcificada selecionada, foi feita a análise dos diagramas de dispersão dos dados 


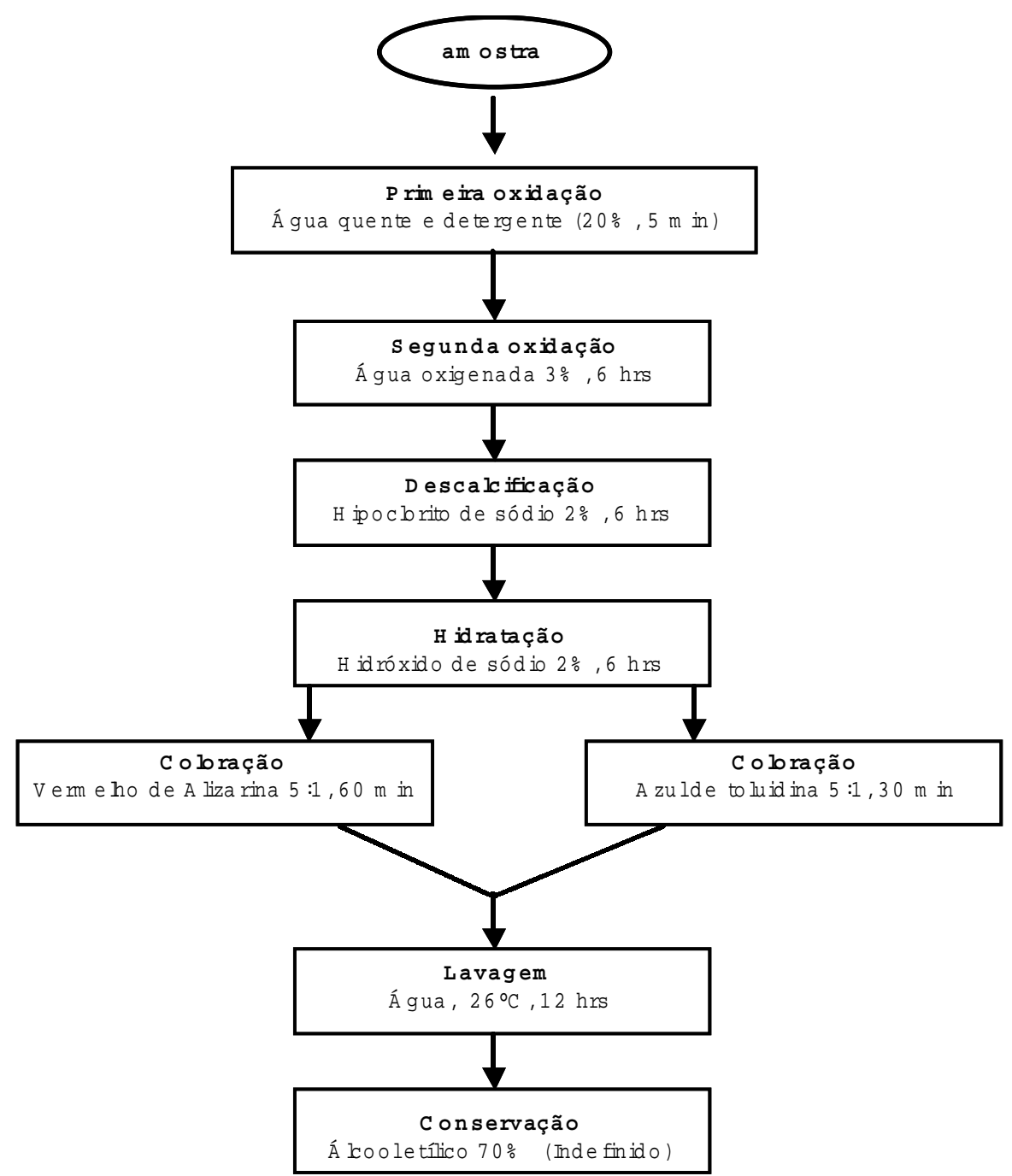

Figura 3 - Fluxograma da técnica de coloração de Hiper-oxidação e Descalcificação Química (HDQ).

considerando-se o total de indivíduos. Em seguida, foram testadas as diferenças entre os valores dos coeficientes de regressão ("b") e dos interceptos da ordenada ("a") utilizando o teste student, com previa verificação da homocedasticidade das variâncias, para detectar diferenças significativas entre machos e fềmeas (ZAR,1996).

Para determinar o período de formação das marcas principais de crescimento, foi calculado o incremento marginal relativo mensal definido por Fabré \& Saint Paul (1998). Fo- ram medidas as distâncias entre o raio total da estrutura selecionada a partir do centro da estrutura até as duas últimas marcas de crescimento e aplicada a expressão:

$\mathrm{IMR}=\left(\mathrm{R}_{\mathrm{t}}-\mathrm{R}_{\mathrm{n}} / \mathrm{R}_{\mathrm{n}}-\mathrm{R}_{\mathrm{n}-\mathrm{-}}\right) \quad$ (Fabré \& Saint Paul, 1998) Onde:

$\mathrm{Rt}=$ raio total da estrutura

$\mathrm{R}_{\mathrm{n}}=$ distância do centro da estrutura até a última marca e

$\mathrm{R}_{\mathrm{n}-1}=$ distância do centro até a penúltima marca. 
O período de formação da marca de crescimento foi considerado como aquele em que houve uma diminuição súbita e um subsequente aumento dos valores do incremento marginal relativo.

Para detectar diferenças significativas entre os valores médios mensais do IMR ao longo do ano, indicativas do período de formação da marca de crescimento na estrutura calcificada, foi utilizado uma análise de variância simples, com prévia verificação da homocedasticidade das variâncias pelo teste de Bartlett e em seguida foi aplicado o teste LSD (Least Significantive Diference, SOKAL \& ROHLF, 1981) de comparações múltiplas para definir as diferenças entre as médias utilizando-se o correspondente nível de confiança $(95 \%)$.

\section{RESULTADOS}

\section{Opérculos}

As observações preliminares dos opérculos demostraram que essas estruturas são muito calcificadas e porosas, portanto, opacas para a visualização de marcas, mesmo após terem sido clarificadas ou coradas. Tanto a técnica de La Marca (1966) como a de Daiber (1960), demonstraram que os opérculos de Calophysus macropterus não apresentaram nenhum tipo de marca que pudesse ser considerada como marca de crescimento.

\section{Otólitos}

As marcas de crescimento nos otólitos asteriscus, foram melhor observadas com benzoato de metil a 24 horas de exposição. Uma exposição maior que 24 horas, cria uma descalcificação completa da estrutura.

$\mathrm{Na}$ análise qualitativa dos 111 otólitos asteriscus observados, mostraram que somente 48 apresentaram marcas visíveis. Demonstrouse que somente $38(34 \%)$ otólitos usando benzoato de metil e 10 (9\%) usando Xilol, mostraram algum tipo de marca visível. Praticamente não há marcas intermediárias, sendo somente encontradas em $8 \%$ dos casos. As marcas de crescimento são simples, porém pouco nítidas. Nos otólitos analisados não foi possível visualizar mais que cinco marcas.

\section{Vértebras}

Observou-se que o número de marcas nas vértebras não tratadas (controle), assim como, naquelas tratadas com as técnicas de Daiber (1960) e Officer et al.,(1995), foi pouco consistente, já que houve alta variabilidade no número de marcas observadas. Além disso, a absorção do corante foi pouco uniforme, resultando em uma coloração deficiente do corpo vertebral.

No caso da nova técnica de Hiperoxidação e Descalcificação Química, foi obtida uma boa definição das marcas inclusive as localizadas próximas à borda. Dos corantes utilizados, o azul de toluidina proporcionou uma melhor identificação das marcas e maior consistência ou estabilidade no número de marcas contabilizadas.

\section{Padrão de marcação}

Nas vértebras foram identificados três tipos de marcas: marcas principais, marcas intermediárias e marcas largas.

Marcas principais: possuem boa nitidez e apresentam-se estreitas e duplas. O espaçamento dessas marcas seguiu o padrão esperado de crescimento em $78 \%$ dos casos, ou seja a diminuição do espaçamento entre as marcas em direção à borda. Marcas intermediárias: essas marcas são simples e menos nítidas. Entre as marcas principais podem ocorrer uma ou duas marcas intermediárias. A partir da quarta marca principal, as marcas intermediárias são ocasionais.

Marcas largas: são duas marcas escuras e largas, a primeira está localizada entre a primeira e a segunda marca principal, é bem conspícua acompanhada por uma marca intermediária ou em alguns casos, apresenta-se como duas marcas duplas grossas. Entre a segunda e a terceira marca principal apresenta-se uma segunda marca larga (Figura 4). 


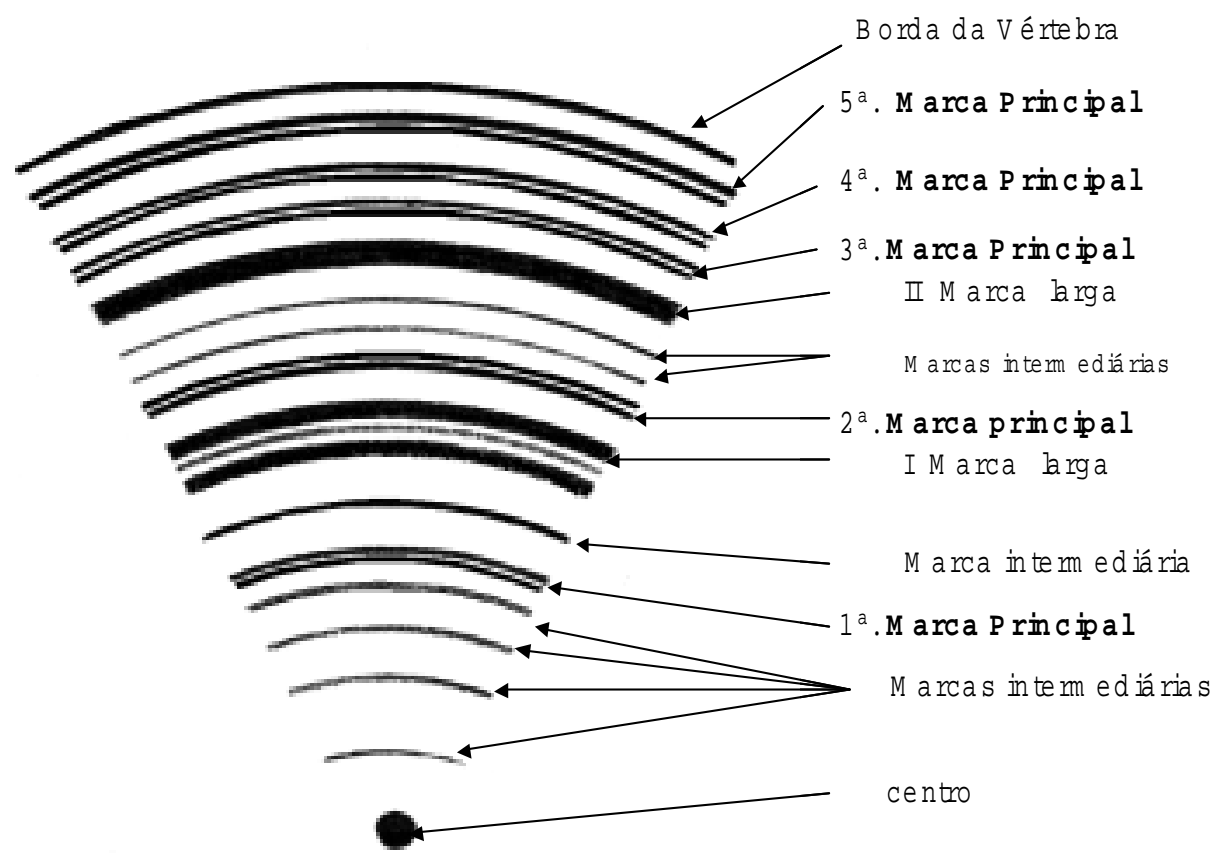

Figura 4 - Desenho esquemático da vértebra de Calophysus macropterus apresentando os diferentes tipos de marcas observadas.

Das vértebras observadas, $60 \%$ apresentaram marcas de crescimento bem nítidas, embora devamos destacar que a presença constante de marcas intermediárias (98\% dos casos) é uma característica dessas estruturas. Outro aspecto constatado, foi a alta porcentagem de marcas principais duplas $(66 \%)$.

Entre o centro da vértebra e a primeira marca principal existe uma zona ampla com duas a três ou excepcionalmente quatro marcas simples. Elas são de ocorrência variável e com diferentes graus de nitidez. Portanto, essas marcas não foram consideradas como indicador da idade.

As dificuldades presentes no processo de leituras destas estruturas foram: a presença de marcas duplas ou múltiplas e a proximidade das marcas. Em alguns casos encontramos uma série de duas marcas principais juntas; quando isto aconteceu, foi dada maior atenção ao padrão esperao de crescimento.

\section{Distribuição das distâncias nas marcas principais}

$\mathrm{Na}$ distribuição de freqüência da distância de cada marca principal desde a origem, observou-se uma moda se deslocando progressivamente (Figura 5), conforme o aumento dos comprimentos médios de cada marca de crescimento. $\mathrm{O}$ distan-ciamento entre a moda de uma marca e a seguinte é menor à medida que se afasta do centro da vértebra e aumenta o número de marcas. Isto indica que o padrão de marcação dos anéis ou as marcas de crescimento nas vértebras de Calophysus macropterus, representou uma diminuição progressiva dos incrementos de crescimento, conforme o aumento da idade.

Ainda, a normalidade verificada no teste Kolmogorov-Smirnov (Sokal \& Rolf, 
1981) e a uni modalidade observadas nas distribuições da primeira à quinta marca principal (Figura 5), indicam que foram mantidos os critérios de identificação de cada marca durante as leituras. A falta de ajuste observado para a sexta e sétima marca principal pode ser atribuído ao baixo número de observações.
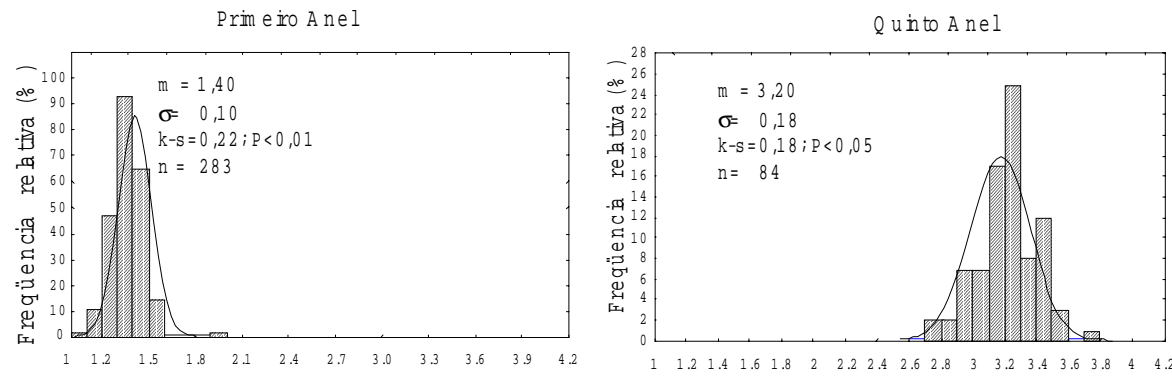

Segundo Anel
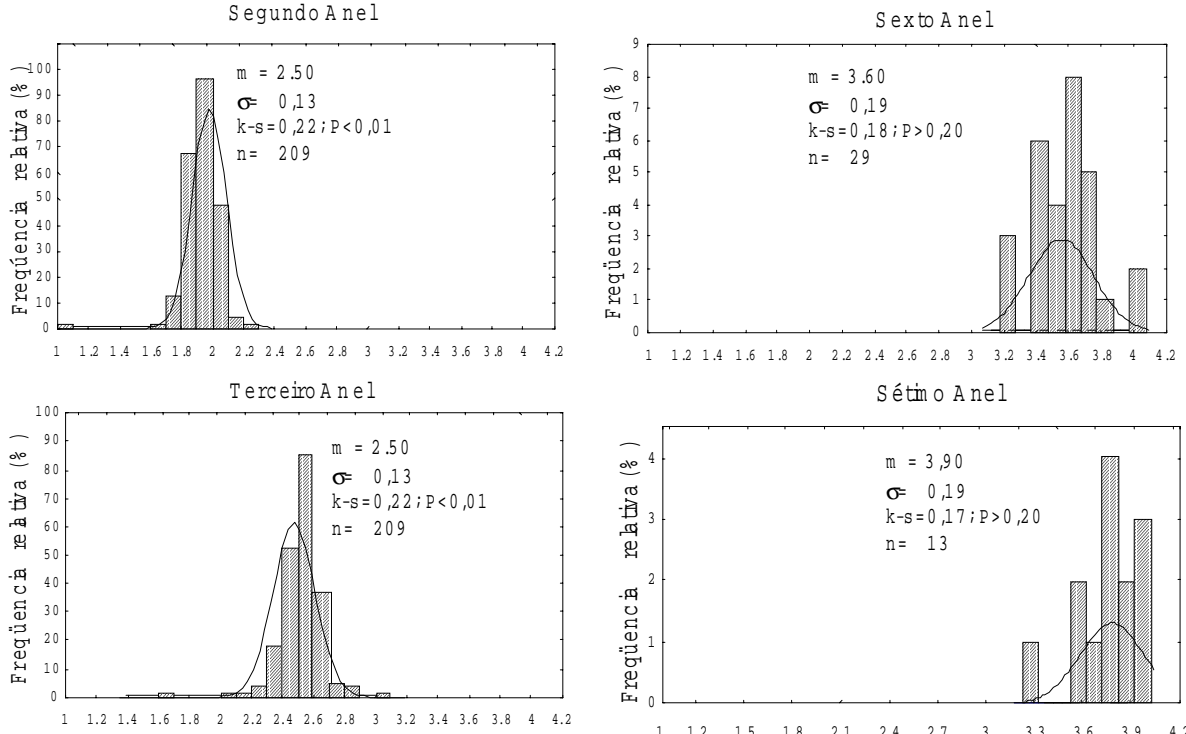

S étín o A nel

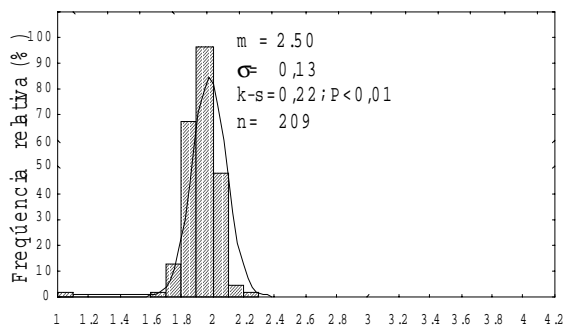

Q uarto A nel
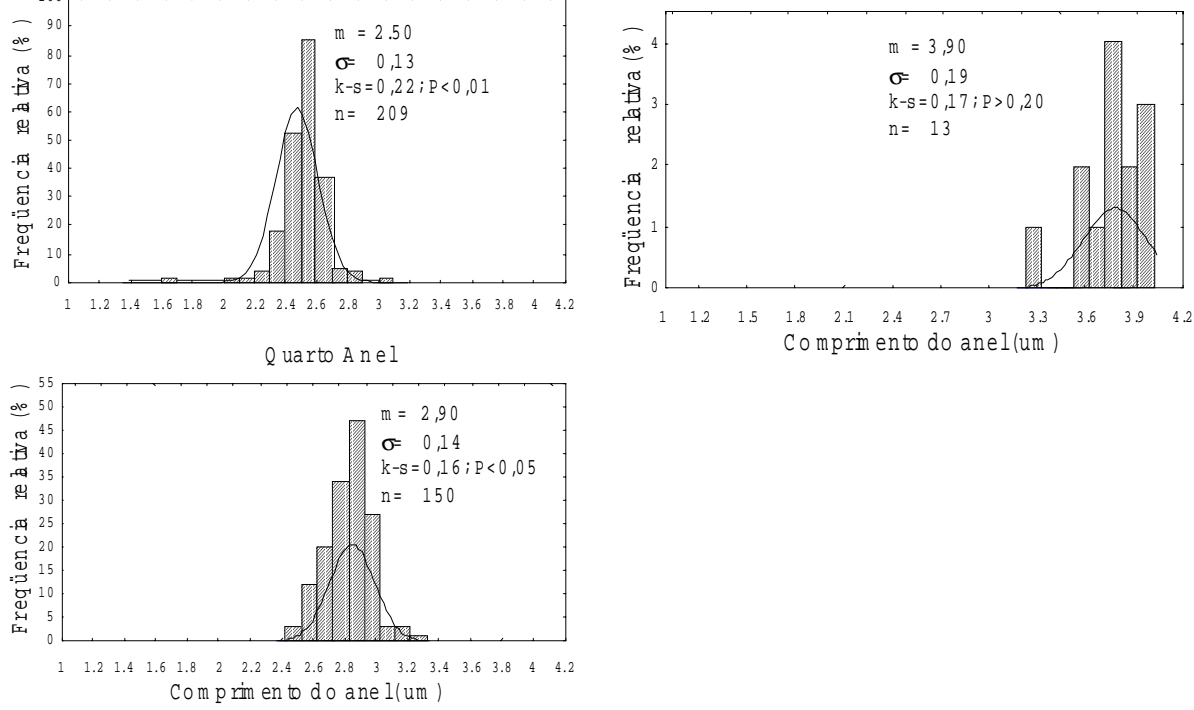

Figura 5 - Distribuição de freqüência do comprimento dos anéis (distância centro-borda), indicando o ajuste à normal pelo teste Kolmogorov-Smirnov $(\mathrm{k}-\mathrm{s})$, com as médias $(\overline{\mathrm{x}})$, desvio padrão $(\sigma)$ e número de observações (n) por marcas principais, nas vértebras de Calophysus macropterus 


\section{Morfometria das estruturas calcificadas}

Todas as medidas realizadas nos otólitos se destacaram por apresentar uma baixa relação corpo-estrutura (tabela 1). Desta forma, devido ao baixo coeficiente de correlação e a dificuldade de visualização das marcas de crescimento, estas estruturas foram descartadas para a determinação da idade no presente trabalho.

Um bom ajuste ao modelo linear foi obtido para a relação entre o comprimento padrão $(\mathrm{Cp})$ e o raio da vértebra $(\mathrm{Rt})$, tanto para o total de indivíduos como para cada sexo (Tabela 2). Por outro lado, não foram observadas diferenças significativas nos valores de "b" (t-student, $\mathrm{t}_{\mathrm{obs}}<1,17$; g. $\left.\mathrm{l}_{(123)}\right)$, nem entre os valores de "a" (t-student, $\left.\mathrm{t}_{\text {obs }}<1,15 ; \mathrm{g.l}_{(123)}\right)$ tanto em fêmeas como em machos. Portanto, o melhor modelo para o total de indivíduos da relação raio vertebral-comprimento padrão é definido pela equação $\mathrm{Cp}=-0,158+0,111 * \mathrm{Rt}$ (Tabela 2).

Para a analise do IMR foi utilizado as vértebras com duas, três e quatro marcas principais. Os valores do IMR apresentaram uma distribuição normal (K-S: $\left.\quad \mathrm{D}_{0.05[123]}=0.10039 ; \quad \mathrm{D}_{\mathrm{obs}}=0.10025\right)$ e a distribuição das variâncias foram homogêneas (Bartlett $\mathrm{x}^{2}{ }_{0.05[11]}=19.675$; $\left.\chi^{2}{ }_{\text {obs }}=16.690\right)$, assim, os dados do IMR cumprem com os pressupostos da ANOVA.

Tabela 1 - Coeficiente de correlação (r), graus de liberdade (g.1.), probalidade (P) e resultado do teste de Fisher $(\mathrm{F})$ das diferentes relações morfométricas entre as diferentes medidas nos raio dos otolitos e o comprimento padrão $(\mathrm{Cp})$ de Calophysus macropterus.

\begin{tabular}{lccccc}
\hline $\begin{array}{l}\text { RELAÇÃO VÉRTEBRA-COMPRIMENTO } \\
\text { Comprimento padrão vs. }\end{array}$ & $r$ & $b$ & $\begin{array}{c}\text { g.l. } \\
\text { v1; v2 }\end{array}$ & $P$ \\
\hline $\begin{array}{l}\text { Distância raio total (Fêmeas) } \\
\text { Distância raio total (Machos) }\end{array}$ & 0.801 & 0.104 & $(1,103)$ & $<0.05$ \\
Distância raio total (Sexos combinados) & 0.905 & 0.105 & $(1,77)$ & $<0.05$ \\
\hline
\end{tabular}

* Sexos combinados

Tabela 2 - Coeficiente de correlação (r), graus de liberdade (g.l.), probalidade (P) e coeficiente de declividade (b) das diferentes relações morfométricas entre as vértebras e o comprimento padrão (Cp) de Calophysus macropterus para fêmeas machos e sexos combinados.

\begin{tabular}{lcccr}
\hline $\begin{array}{l}\text { RELAÇÃO VÉRTEBRA-COMPRIMENTO } \\
\text { Comprimento padrão vs. }\end{array}$ & $r$ & $b$ & $\begin{array}{c}\text { g.l. } \\
\text { v1; v2 }\end{array}$ & $P$ \\
\hline $\begin{array}{l}\text { Distância raio total (Fêmeas) } \\
\text { Distância raio total (Machos) }\end{array}$ & 0.801 & 0.104 & $(1,103)$ & $<0.05$ \\
& 0.905 & 0.105 & $(1,77)$ & $<0.05$ \\
Distância raio total (Sexos combinados) & 0,915 & 0.111 & $(1,122)$ & $<0,05$ \\
\hline
\end{tabular}


A análise de variância, demonstrou que existem diferenças significativas entre os meses, indicando uma diminuição significativa no IMR e consequentemente no ritmo de crescimento sazonal. O teste LSD revelou que entre os meses de julho e agosto e entre janeiro e fevereiro, existe uma queda significativa $(\mathrm{P}<0,05)$ nos valores do incremento marginal relativo (IMR) médios (Tabela 3 ).

Os resultados do IMR relacionados com o ciclo hidrólogico do rio, indicam que esta espécie forma duas marcas de crescimento por ano, uma no mês de janeiro durante a enchente e outra no mês de julho, período de vazante na região.

Tabela 3 - Resultados do teste Least Significantive Diference (LSD) aplicado os valores do IMR da Piracatinga (Calophysus macropterus), considerando o mês como fator

\begin{tabular}{|c|c|c|c|c|c|c|c|c|c|c|c|c|}
\hline MÊS & jan & fev & mar & $a b r$ & mai & jun & jul & ago & set & set & nov & dez \\
\hline MEDIA & 81,243 & 57,227 & 58,308 & 62,519 & 52,259 & 45,415 & 48,074 & 26,610 & 53,895 & 64,411 & 58,490 & 72,366 \\
\hline jan & & 0,0099 & 0,0043 & 0,0219 & 0,0011 & 0,0003 & 0,0003 & 0,0000 & 0,0010 & 0,0838 & 0,0035 & 0,3263 \\
\hline fev & 0,0099 & & 0,8880 & 0,5005 & 0,5590 & 0,2138 & 0,2993 & 0,0011 & 0,6739 & 0,4489 & 0,8651 & 0,0869 \\
\hline mar & 0,0043 & 0,8880 & & 0,5043 & 0,3942 & 0,1191 & 0,1711 & 0,0001 & 0,4898 & 0,4595 & 0,9749 & 0,0607 \\
\hline$a b r$ & 0,0219 & 0,5005 & 0,5043 & & 0,1598 & 0,0431 & 0,0597 & 0,0000 & 0,1917 & 0,8219 & 0,5022 & 0,1980 \\
\hline mai & 0,0011 & 0,5590 & 0,3942 & 0,1598 & & 0,4479 & 0,6141 & 0,0037 & 0,8239 & 0,1786 & 0,3620 & 0,0163 \\
\hline jun & 0,0003 & 0,5590 & 0,1191 & 0,0431 & 0,4479 & & 0,7750 & 0,0536 & 0,3172 & 0,0574 & 0,1042 & 0,0042 \\
\hline jul & 0,0003 & 0,2993 & 0,1711 & 0,0597 & 0,6141 & 0,7750 & & 0,0184 & 0,4501 & 0,0803 & 0,1495 & 0,0053 \\
\hline ago & 0,0000 & 0,0011 & 0,0001 & 0,0000 & 0,0037 & 0,0536 & 0,0184 & & 0,0010 & 0,0001 & 0,0001 & 0,0000 \\
\hline set & 0,0010 & 0,6739 & 0,4898 & 0,1917 & 0,8239 & 0,3172 & 0,4501 & 0,0010 & & 0,2152 & 0,4508 & 0,0174 \\
\hline set & 0,0838 & 0,4489 & 0,4595 & 0,8219 & 0,1786 & 0,0574 & 0,0803 & 0,0001 & 0,2152 & & 0,4605 & 0,3928 \\
\hline nov & 0,0035 & 0,8651 & 0,9749 & 0,5022 & 0,3620 & 0,1042 & 0,1495 & 0,0001 & 0,4508 & 0,4605 & & 0,0554 \\
\hline dez & 0,3263 & 0,0869 & 0,0607 & 0,1980 & 0,0163 & 0,0042 & 0,0053 & 0,0000 & 0.0174 & 0,3928 & 0,0554 & \\
\hline
\end{tabular}

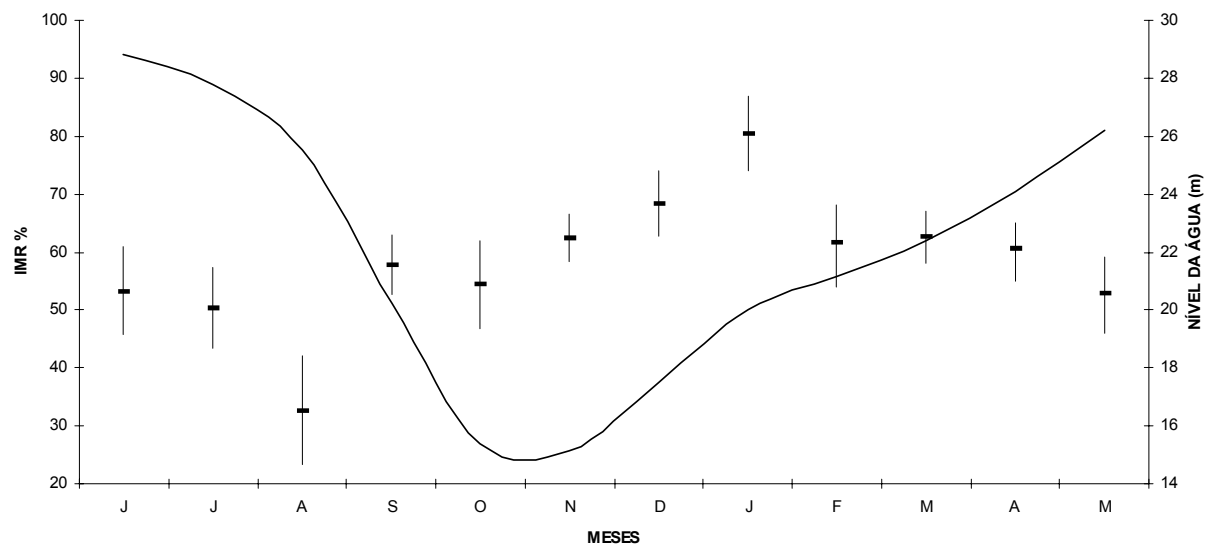

Figura 6. Valores médios mensais do incremento marginal relativo (IMR) das marcas principais nas vértebras de Calophysus macropterus, com os intervalos de confiança LSD $(\propto=0,05)$ e a variação no nível do rio (linha continua), de junho/97 a maio/98. 
Quando foram analisadas as freqüências de ocorrência dos valores do IMR agrupados em intervalos de classes de $30 \%$, verificou-se que IMR superior a $50 \%$ são freqüentes entre junho e julho, entretanto, há um deslocamento dos valores modais $(<50 \%)$ para a esquerda em agosto. Após este período, incrementa-se a ocorrência de valores altos $(>50 \%)$, resultando em um deslocamento modal da distribuição de freqüência para a direita (Figura 7).

Entre dezembro e janeiro foram observados indivíduos com IMR maiores que $100 \%$, indicando que possivelmente existe uma marca na borda que só pode ser observada a partir de fevereiro ou março, quando aumenta novamente o ritmo de crescimento e o conseqüente deslocamento da distribuição de freqüência para a direita mantendo-se este padrão até maio (Figura 7).

A análise dos histogramas de freqüência relativa concorda com o resultado da análise de variância, demostrando também, que a época de formação das marcas de crescimento ocorre na vazante (agosto) e na enchente (janeiro), (Figura 7).

\section{DISCUSSÃO}

A precisão nos estudos de determinação de idade e crescimento através da interpretação e contagem de marcas nas estruturas aumenta quando são analisadas e comparadas diferentes estruturas calcificadas (Casselman,1983; Brothers,1983). No caso de Calophysus macropterus, foram analisadas três tipos de estruturas de aposição freqüentemente utilizadas nos estudos de determinação de idade: opérculos, otólitos e vértebras.

Os otólitos têm sido freqüentemente utilizados para a determinação da idade de espécies de peixes Siluriformes de clima tropical (Fabré et al., 1995; Muñoz-Sosa 1996; Valeruz et al., 1998). Não obstante, os resultados deste trabalho indicam que o otólito asteriscus de Calophysus macropterus após o tratamento com benzoato de metil, apresentouse ainda opaco, portanto, as marcas não puderam ser claramente identificadas.

As principais dificuldades encontradas na análise desta estrutura foram: indefinição da primeira marca, presença de extensas zonas opacas que dificultaram a observação dos anéis hialinos e a baixa correlação entre o comprimento padrão do peixe e o tamanho do otólito. Estas dificuldades podem ser atribuídas às características próprias das estruturas ou à aplicação de técnicas não adequadas para a conservação e/ou observação do otólito asteriscus .

Com as técnicas de Daiber (1960), La Marca (1966) e Officer et al. (1995), não verificamos um padrão de marcação de anéis duplos estáveis nas vértebras. Porém, quando aplicamos a técnica de Hiperoxidação e Descalcificação Química, nas vértebras, foi possível distinguir as marcas principais das marcas intermediárias. Adicionalmente se verificou uma relação linear entre o raio da estrutura com o comprimento padrão da piracatinga.

Nas vértebras de Calophysus macropterus as marcas intermediárias entre as marcas principais podem confundir a contagem. Panfili (1993) e Villacorta-Corrêa (1997) não conseguiram observar um padrão de marcação nas vértebras de Colossoma macropomum, ocasionado principalmente pela alta freqüência de ocorrência de marcas intermediárias. No caso das vértebras de Calophysus macropterus, o critério da redução progressiva da distância (padrão esperado de crescimento) entre as marcas principais e sua marcação dupla constante foi importante para a identificação de um padrão fixo de marcação.

Com nenhuma das técnicas de clarificação (Daiber,1960) ou de coloração (La Marca, 1966) utilizadas foi possível identificar marcas de crescimento nos opérculos. Portanto, consideramos que esta estrutura não é adequada para determinar a idade em Calophysus macropterus. Isto também foi observado por Valeruz et al., (1998) em Brachyplatystoma flavicans e por Panfili (1993) em Pseudoplatystoma fasciatum. Estas estruturas podem não ser indicadas para estudos de determinação de idade em bagres, possivelmente, por estarem constituídas por tecido ósseo esponjoso, poroso não laminar e pouco translúcido, fato que foi constatado neste estudo.

No caso da piracatinga, a avaliação da idade através da comparação em diferentes 

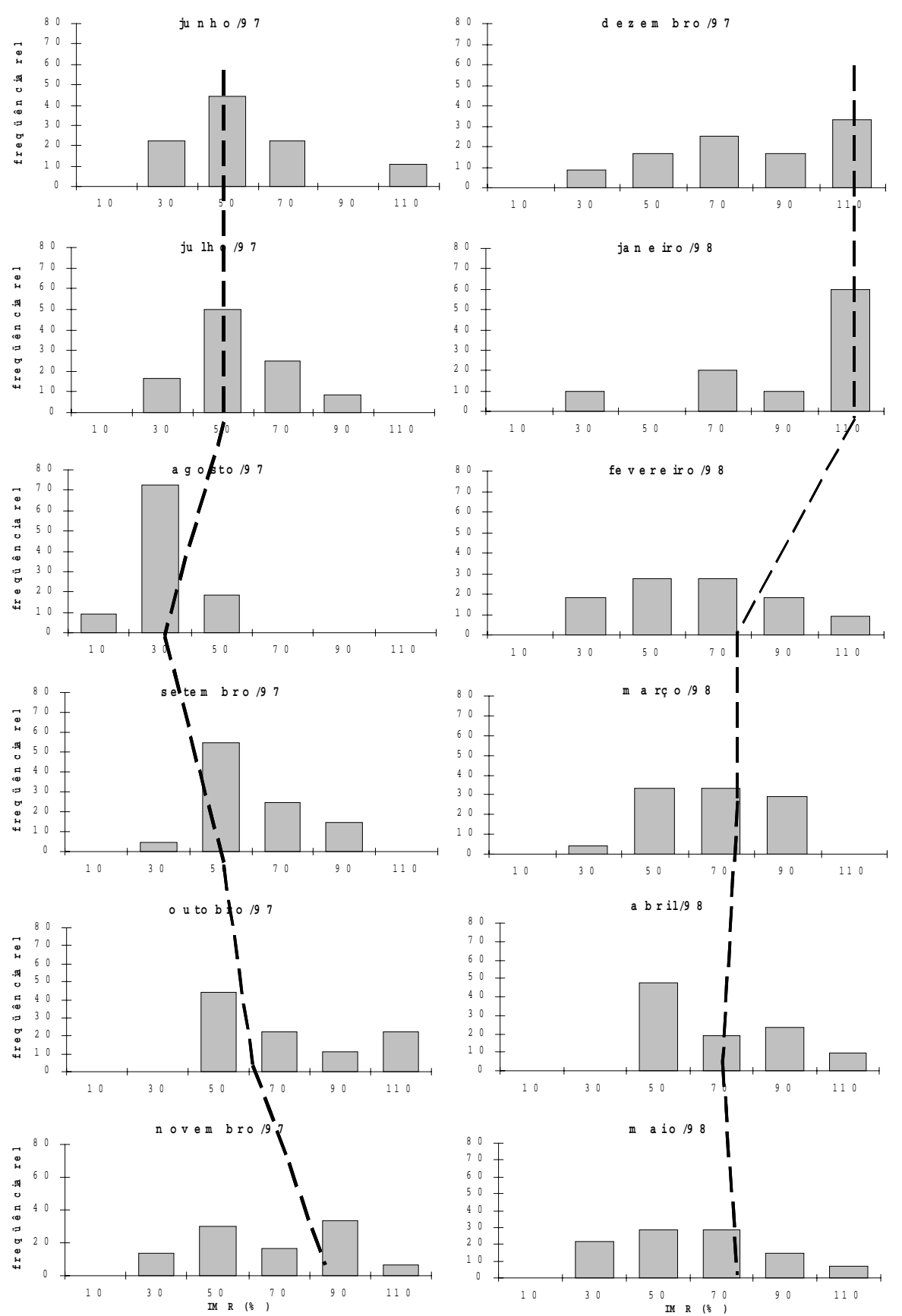

Figura 7. Distribuição mensal da freqüência relativa do incremento marginal (IMR), observado nas análises das vértebras de Calophysus macropterus entre junho/97 a maio/98, a linha tracejada indica o deslocamento das freqüências. 
estruturas calcificadas do mesmo peixe (verificação), não foi possível de realizar devido ao fato de somente uma das três estruturas calcificadas analisadas, apresentou anéis de crescimento visíveis.

As técnicas tradicionais de coloração de vértebras, caracterizam-se por iniciar-se com uma oxidação após a coloração ou pela descalcificação antes da coloração. Mas o problema com estas técnicas é que a visualização das marcas torna-se difícil pela presença de tecido adiposo, conectivo e sangue, devido à dificuldade da sua extração do tecido ósseo poroso das vértebras.

$O$ resultado obtido neste trabalho aplicando a técnica de Hiper-oxidação e Descalcificação Química, mostra o grande potencial desta técnica que pode ser utilizada em estruturas calcificadas de outras espécies. Destacamos que para sua aplicação, esta técnica somente serve para estruturas calcificadas constituídas por tecidos ósseos de tipo laminar e não porosa, como os opérculos da própria piracatinga (Calophysus macropterus) e da dourada (Brachyplatystoma flavicans)

Apesar da necessidade de aplicar um tratamento elaborado nas vértebras para obter uma coloração uniforme, fato que demanda muito tempo, esta metodologia é vantajosa porque, além de permitir a distinção das marcas de crescimento, não exige muito cuidado no momento da extração da estrutura, como no caso dos otólitos. Desta forma, a vértebra por apresentar um padrão de marcação estável de anéis indicadores de crescimento e um bom ajuste na relação corpo/estrutura, foi selecionada como a estrutura calcificada mais adequada para a determinação da idade da piracatinga, indicando que estas estruturas podem ser utilizadas em estudos de idade desta espécie.

Na piracatinga foi verificada a formação de duas marcas por ano: um durante a vazante e outro durante a enchente, resultado também observado em alguns Characiformes tais como: Prochilodus nigricans (Oliveira, 1997); Brycon cephalus (Villacorta-Corrêa, 1987) e Semaprochilodus insignis (Vieira, 1999). Esta periodicidade de marcação de Calophysus macropterus difere da encontrada em outros bagres para os quais a formação das marcas indicativas da idade é anual (Muñoz-Sosa,
1996; Panfili, 1993; Reid, 1983; Reina et al. 1995).

Esta diferença na periodicidade de marcação poderia ser explicada pela dificuldade de interpretação das marcas nas vértebras, influenciando o resultado da validação. Welcomme (1992) relata que nas estruturas calcificadas dos peixes tropicais têm sido registradas mais marcas do que seria esperado, mostrando que a formação das marcas não depende de um único evento regular sazonal.

\section{AGRADECIMENTOS}

Nosso agradecimento para Vandick Batista que gentilmente cedeu o laboratório de investigações pesqueiras da Universidade do Amazonas, à Elisabeth Vieira pela revisão do português, ao Instituto Nacional de Pesquisas da Amazônia (INPA) e ao CNPq através do projeto: Avaliação da Pesca e Dinâmica Populacional dos Recursos Pesqueiros da Amazônia Central, INPA-FUA-PTU-CNPq, pelo suporte financeiro.

\section{BIBLIOGRAFIA CITADA}

Barthem, R. 1990. Ecologia e Pesca da Piraiba (Brachyplatystoma vaillantii). Tese de Doutorado Universidade Estadual de Campinas. São Paulo. SP 268p.

Beamish, R.J. 1981. Use of fin-ray section to age Walleye Pollock, Pacific Cod, and the importance of this method. Transantions of the American Fisheries Society, 100:287-299.

Beamish, R.J.; Clinton, D. 1977. Age determination of lingcod (Ophiodon elongatus) using dorsal fin rays and scales. Journal Fisheries Research Board Canadian, 34:1305-1313.

Brothers, E. B. 1983. Summary of round table discusion on age validation. In: Prince, E. D.; Pulos, L. M. (Ed.). Proceeding the international workshop on age determination of oceanic pelagic fishes. Tunas and Sharks. NOAA Technical report, 8, National Marine Fisheries Service, USA. $35-44 p$. 
Casselman, J.M. 1983. Age and growth assessment of fish from their calcified structures techniques and tools. In: Prince, E.D.; Pulos, L.M. (Ed.). Proceeding the international workshop on age determination of oceanic pelagic fishes. Tunas and Sharks. NOAA Technical report, 8, National Marine Fisheries Service, USA $1-17 p$.

Daiber, F.C. 1960 A technique for age determination in the skate Raja eglanteria. Copeia, (3): 258-260.

Fabré, N.N.; Gomes, A.L.S.; Batista, V.S. 1995. Determinação de idade em siluriformes amazônicos. I análise de estruturas na Piracatinga Callophysus macropterus . XI Encontro Brasileiro de Ictiologia, Campinas, SP. 3p.

Fabré, N.N; Saint-Paul, U.1998. Annulus formation on scales and seasonal growth of the Central Amazonian anostomid Schizodon fasciatus. Journal of Fish Biology, 53: 1-11.

La Marca, M.J. 1966. A simple technique for demonstrating calcified annuli in the vertebrate of large elasmobranchs. Copeia, (2): 351-352.

Muñoz-Sosa, D.L. 1996. Age and structure and exploitation of giant catfish populations (Brachyplatystoma spp.) in the Lower Caqueta river, Colombia. Master of Science thesis. State University of New York, Syracusa, USA. 101p.

Nikolsky, G.V.1969. Theory of the fish population dynamics as the biological background for rational exploration and management of fishery resources. Oliver \& Boyd, London. 323p.

Officer, R.A; Gason, A.S.; Walker, T.I.; Clement, J. G. 1995. Sources of the variation in the counts of growth increments in vertebrae from gummy shark, Mustelus antarticus, and school shark, Galeorhinus galeus: implications for age determination. Canadian Journal Aquatics Sciences, 53:1765-1777.

Oliveira, I.1997. Determinação da idade e aspectos da dinâmica populacional do Curimatã Prochilodus nigricans (Pisces:
Prochilodontidae) da Amazônia Central . Dissertação de Mestrado. Instituto Nacional de Pesquisas da Amazônia/ Fundação Universidade do Amazonas. Manaus, Amazonas. 90p.

Panfili, J. 1993. Estimation de age individuel des poissons: methodologies et applications á des populations naturelles tropicales et tempérées. Diplome de Doctorat. Université de Monpellier, France. 456p.

Reid, S. 1983. La biología de los bagres rayados Pseudoplatystomas fasciatus y P. tigrinus. En la Cuenca del rio Apure, Venezuela. Revista UNELLEZ de Ciencia y Tecnologia, 1(1):13-41.

Reina, M.; Ramirez, H.; Valderrama, M. 1995. Edad y crecimiento de Paulicea lutkeni (Steindacher, 1876) (PISCES: PIMELODIDADE) basado en la lectura de estructuras duras (espinas de la aleta pectoral) en el alto rio Meta (Colombia).Boletin cientifico INPA, No.3 115-130.

Sokal, R. R.; Rohlf, F. J. 1981. Biometry: The principles and practice of statistics in biological research. W.H. Freeman \& Co. Oxford, USA.

Villacorta-Corrêa, M.1987. Crescimento do matrinxã, Brycon cephalus (Gunther, 1969) (teleostei:Characidae) no baixo rio negro, seus afluentes e no baixo Solimões. (Dissertação de Mestrado), Instituto Nacional de Pesquisas da Amazônia/ Fundação Universidade do Amazonas. Manaus, Amazonas. 124p.

Villacorta-Correa, M.1997. Estudo de idade e crescimento do Tambaqui Colossoma macropomum Characiformes: Characidae no amazonas central, pela analise de marcas sazonais nas estruturas mineralizadas e microestruturas nos otólitos. Tese de Doutorado. Instituto Nacional de Pesquisas da Amazônia/ Fundação Universidade do Amazonas. Manaus, Amazonas. 216p.

Valeruz-Rego, H.V.; Fabré, N.N.; Pérez, A. 1998. Avaliação de estruturas calcificadas de dourada Brachyplastystoma flavicans 
(Pisces: Pimelodidae) para a determinação da idade na Amazônia Central. Boletim do Museu Paraense Emílio Goeldi. ser. zool., 14(2): 143-173.

Vieira, E. F. 1999. Determinação da idade e crescimento do jaraqui de escama grossa (Semaprochilodus insignis) na Amazônia Central. Dissertação de mestrado, Instituto Nacional de Pesquisas da Amazônia/Fundação Universidade do Amazonas. Manaus, Amazonas. 86p
Welcomme, R. L.1992. Pesca fluvial. FAO, Documento Técnico de Pesca 262. Roma. 303p.

Werder, U.; Soares, G. 1985. Age determination by sclerite numbers, and scale variantions in six fish species from the Central Amazon (Osteichthyes; Characoidei). Amazoniana, 8(3): 395-420.

Zar, J.H. 1996. Biostatistical analysis. 3rd. Edition. Prince-Hall Inc., New Jersey. $662 \mathrm{p}$. 
